\title{
Ultra-low material loss microstructure fiber for terahertz guidance
}

\author{
Md. Rabiul Hasan, ${ }^{* 1}$ S. Ali $^{2}$ and S. A. Emi ${ }^{1}$ \\ ${ }^{I}$ Department of Electronics \& Telecommunication Engineering, Rajshahi University of Engineering \& Technology, \\ Rajshahi 6204, Bangladesh \\ ${ }^{2}$ School of Computer and Communication Engineering, Center of Excellence, Advanced Communication \\ Engineering Cluster, Pauh Putra Campus, Arau 02600, Perlis, Malaysia
}

Received October 25, 2016; accepted March 08, 2017; published June 30, 2016

\begin{abstract}
In this letter, we numerically demonstrate a hybrid-core microstructure fiber for low-loss terahertz guidance. A finite element method with circular perfectly matched layer boundary conditions is applied to characterize the guiding properties. It is shown that by using a triangular-core inside a square lattice microstructure exhibits an ultralow effective material loss (EML) of $0.169 \mathrm{~dB} / \mathrm{cm}$ and a low confinement loss of $0.087 \mathrm{~dB} / \mathrm{cm}$ at an operating frequency of 0.75 THz. We also discuss how other guiding properties including power fraction, single mode propagation and dispersion vary with the changing of core diameter and operating frequencies. This low-loss microstructure fiber can be effectively used in numerous applications in the $\mathrm{THz}$ regime.
\end{abstract}

The terahertz $(\mathrm{THz})$ radiation spectrum, which occupies the frequency range from 0.1 to $10 \mathrm{THz}$ has gained enormous attention in recent years. This narrow spectrum has been utilized in several promising applications including medical imaging, sensing, biotechnology, homeland security and spectroscopy [1]. Most $\mathrm{THz}$ application systems are based on planar or curved metal reflectors, which are bulky and severely affected by environmental disturbance. Therefore, it is essential to have an extremely low-loss $\mathrm{THz}$ waveguide for a flexible and compact $\mathrm{THz}$ system.

Researchers have already explored several $\mathrm{THz}$ waveguides such as metallic wires, dielectric metal-coated tube, metallic slot waveguide and bare metal wires [2-3]. These waveguides suffer specifically from such issues as strong dispersion near the cut-off frequency, external perturbation with environment etc. Recently, porous-core photonic crystal fibers (PC-PCFs) have attracted much attention because they exhibit low effective material loss (EML), low confinement loss $\left(\alpha_{\mathrm{CL}}\right)$ and low dispersion at $\mathrm{THz}$ frequency bands. Hexagonal PC-PCF have been proposed in [4] having an EML of $0.286 \mathrm{~dB} / \mathrm{cm}$ at $1 \mathrm{THz}$. Besides, PC-PCFs based on octagonal cladding reported in [5-6], where EMLs of 0.243 and $0.3 \mathrm{~dB} / \mathrm{cm}$ have been obtained at $1 \mathrm{THz}$, respectively. A square lattice based PC-PCF has been reported [7] having an EML of 0.33 $\mathrm{dB} / \mathrm{cm}$. Recently, the authors have presented very similar works with different core-cladding geometry [8-9]. However, the reported EML was comparatively higher

\footnotetext{
*E-mail: ruetrabu@gmail.com; rabiul.hasan@ ruet.ac.bd
}

about 0.386 and $0.562 \mathrm{~dB} / \mathrm{cm}$, respectively. The structures proposed in [4-9] exhibit a relatively higher EML; therefore, for efficient $\mathrm{THz}$ guidance it is required to design an extremely low-loss waveguide.

In this letter, we propose a new design that consists of square lattice air holes in the cladding whereas the core is formed by the combination of a triangular lattice and circular air-hole rings. Compared to other designs, the proposed structure shows more compactness in the core region resulting in higher core porosity. As a result, a significantly lower EML can be found in our reported porous core fiber than that obtained in [8-9].The proposed PC-PCF shows an extremely low EML of 0.169 $\mathrm{dB} / \mathrm{cm}$ at $0.75 \mathrm{THz}$, which can be obtained by properly choosing the design parameters.

The schematic of the proposed PC-PCF with an enlarged view of the core is shown in Fig. 1. The diameter of the core is denoted by $D_{\text {core }}$. The cladding consists of square lattice air-holes having a hole-to-hole distance of $\Lambda_{1}$ and a diameter of $d_{1}$. The value of $\Lambda_{1}, d_{1}$ is $0.4 D_{\text {core }}$ and $0.92 \times \Lambda_{1}$, respectively.

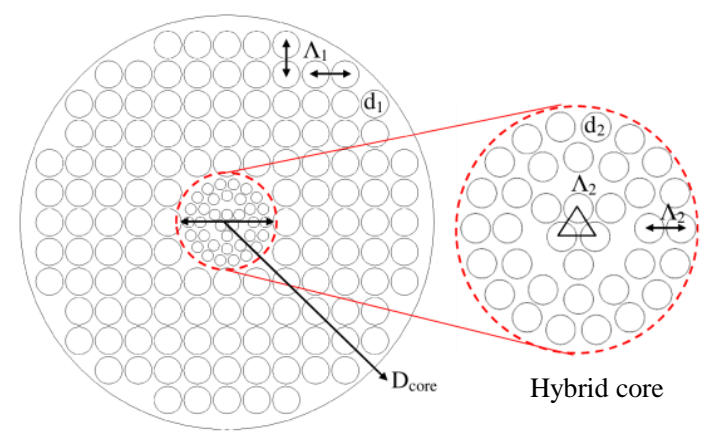

Fig. 1. Cross-section view of the proposed PC-PCF.

The outer-core consists of two circular lattice rings having 12 and 18 air-holes in the $1^{\text {st }}$ and $2^{\text {nd }}$ ring, respectively. There are 6 circular air holes in the inner-core, which form a triangular arrangement. A common pitch $\Lambda_{2}$ is used in the entire hybrid-core, where $\Lambda_{2}=0.14 D_{\text {core }}$. The diameter of all air-holes in the core is $d_{2}$, which is determined by the value of core porosity. Porosity is the ratio between the areas of all air holes in the core to the 
total core area. A cyclic olefin copolymer commercially known as Topas is used as the background material. In the simulation, the bulk absorption loss of Topas has been considered to be $0.9 \mathrm{~dB} / \mathrm{cm}$ at $0.75 \mathrm{THz}$ [10]. The proposed PCF can be fabricated by using either extrusion or capillary stacking techniques.

A finite element method (FEM) based COMSOL v.4.2 software with a circular perfectly matched layer (PML) has been used to simulate the proposed structure. A convergence test has been carried out to adjust the thickness of PML, which was $15 \%$ of the total fiber radius. In the simulation, we have used the following design parameters: $D_{\text {core }}=275 \mu \mathrm{m}, \Lambda_{1}=110 \mu \mathrm{m}, d_{1}=101.2$ $\mu \mathrm{m}, \Lambda_{2}=38.7 \mu \mathrm{m}, d_{2}=41 \mu \mathrm{m}$ and porosity $=80 \%$. Several subsequent simulations have been performed in order to finally adjust these parameters.

A fundamental mode field profile of the proposed PCPCF for different value of core porosities is shown in Fig. 2 at $0.75 \mathrm{THz}$. It can be evident from the figure that electric field distributions are tightly confined in the porous-core region, which permits to lower the EML.
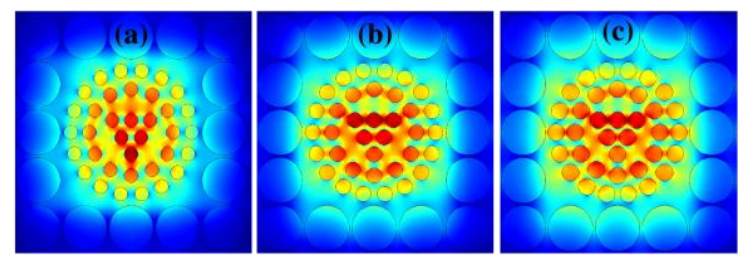

Fig. 2. Mode field profile for (a) $60 \%$, (b) $70 \%$ and (c) $80 \%$ core porosity at $D_{\text {core }}=275 \mu \mathrm{m}$ and $f=1 \mathrm{THz}$

EML is the main loss mechanism of the PC-PCF and can be expression by the perturbation theory in [11],

$$
\alpha_{\text {eff }}=\frac{\left(\varepsilon_{0} / \mu_{0}\right)^{1 / 2} \int_{A_{\text {mat }}} n \alpha_{\text {mat }}|\mathrm{E}|^{2} d \mathrm{~A}}{\left|\int_{A l l} S_{z} d \mathrm{~A}\right|},
$$

where $\varepsilon_{0}$ and $\mu_{0}$ are the permittivity and permeability of vacuum, $\alpha_{\text {mat }}$ is the bulk material absorption loss, $n$ is the refractive index of Topas, and $S_{z}$ is the $z$-component of the Poynting vector. $\alpha_{C L}$ exists in a PC-PCF due to a finite number of air holes used in the cladding and given by the following expression $[4,6,7]$,

$$
\alpha_{C L}=8.686 \cdot \frac{2 \pi f}{c} \operatorname{Im}\left(n_{e f f}\right),
$$

where $f$ is the operating frequency, $c$ is the speed of light in vacuum, and $\operatorname{Im}\left(\mathrm{n}_{\text {eff }}\right)$ is the imaginary part of the effective refractive index. Surface roughness scattering loss is mainly originated from the fiber nonuniformity, which can be effectively reduced by careful fiber fabrication [12]. The scattering loss is inversely proportional to the operating wavelength. At $\mathrm{THz}$ frequencies the induced scattering loss does not significantly affect the total loss [12]. Therefore, we have not considered the scattering loss in our calculation.

Figure 3 shows the variation of EML and $\alpha_{C L}$ as a function of $D_{\text {core }}$ for different core porosities. It is evident that EML can be reduced by using higher core porosity. This is because when core porosity is increased the associated EM field propagates mostly in the core air holes. On the contrary, an opposite trend can be found for the $\alpha_{C L}$ in the same figure. It can be seen that $\alpha_{C L}$ decreases with scaling down of core porosity. Decreasing the core porosity expands the index contrast between core and cladding, which leads to reduced $\alpha_{\mathrm{CL}}$. We have found an EML of $0.169 \mathrm{~dB} / \mathrm{cm}$ and $\alpha_{\mathrm{CL}}$ of $0.087 \mathrm{~dB} / \mathrm{cm}$ at 0.75 THz.

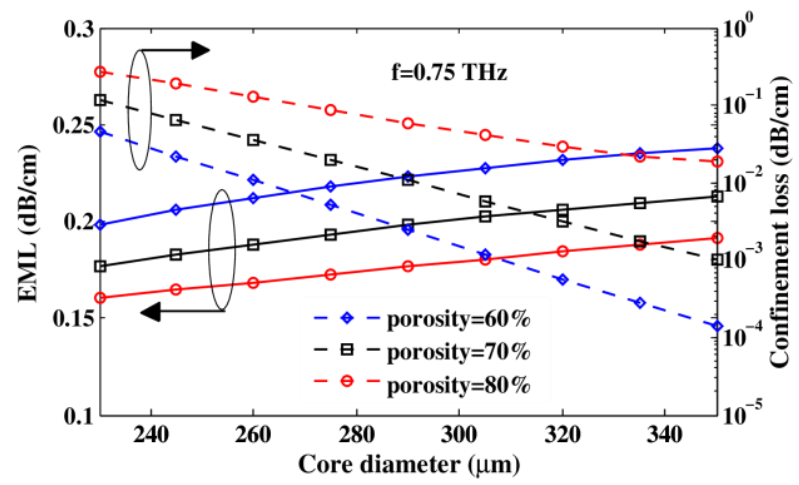

Fig. 3. EML and $\alpha_{\mathrm{CL}}$ as a function of $D_{\text {core }}$ for different core porosities.

Power fraction is the quantity that describes the amount of total power propagates in various regions of the PC-PCF. Power fraction in air-core, air-cladding and background material of the proposed fiber for the variation of $D_{\text {core }}$ are illustrated in Fig. 4. We have found about $34 \%$ of the total power propagates in the core air holes at $D_{\text {core }}=275 \mu \mathrm{m}$.

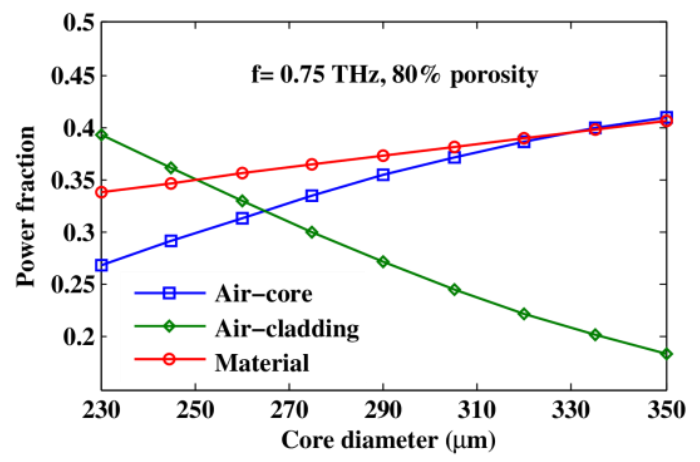

Fig. 4. Power fraction in air-core, air-cladding and material as a function of $D_{\text {core }}$ at $f=0.75 \mathrm{THz}$ and $80 \%$ porosity.

The single mode propagation of the proposed fiber has been investigated based on the confinement loss of higher order modes (HOMs). We have considered the first three core guided modes, which are labeled in LP notation as $\mathrm{LP}_{01}, \mathrm{LP}_{11}$, and $\mathrm{LP}_{21}$. Here, $\mathrm{LP}_{01}, \mathrm{LP}_{11}$, and $\mathrm{LP}_{21}$ are the fundamental mode, first HOM and second HOM, 
respectively. The confinement loss of these modes with a variation of frequency is shown in Fig. 5. It is clearly visible that $\mathrm{LP}_{11}$, and $\mathrm{LP}_{21}$ modes exhibit a comparatively higher confinement loss than that of $\mathrm{LP}_{01}$ mode. As a result, the HOMs will be attenuated rapidly after propagating a short distance. Therefore, it can be concluded that the proposed fiber effectively acts as a single mode fiber between $0.45-1.15 \mathrm{THz}$.

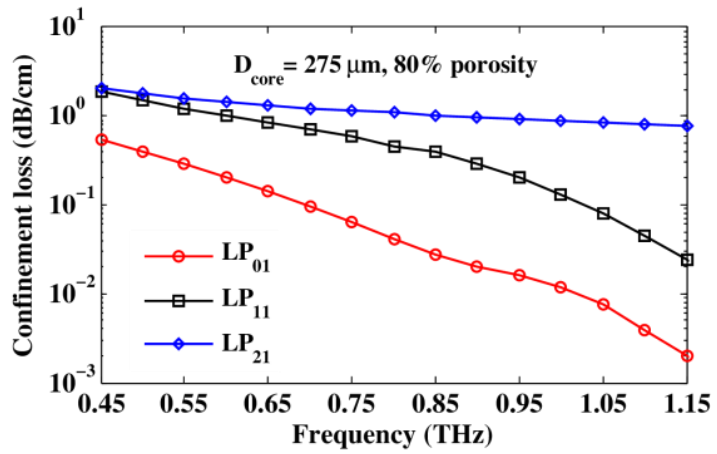

Fig. 5. Confinement loss of the first three guiding modes for $D_{\text {core }}=275 \mu \mathrm{m}$ and $80 \%$ porosity.

Dispersion analysis is important since it represents the amount of pulse spreading when an EM wave propagates certain distances. Topas exhibits a constant refractive index between $0.1-2 \mathrm{THz}$ [10]. As a result, the induced material dispersion can be assumed to be zero. Therefore, the main contribution of the total dispersion is waveguide dispersion $\left(\beta_{2}\right)$, which is given by [13],

$$
\beta_{2}=\frac{2}{c} \frac{d n_{e f f}}{d \omega}+\frac{\omega}{c} \frac{d^{2} n_{e f f}}{d \omega^{2}},
$$

where $n_{\text {eff }}$ is the effective index of core and $\omega$ is the angular frequency $(\omega=2 \pi f)$.

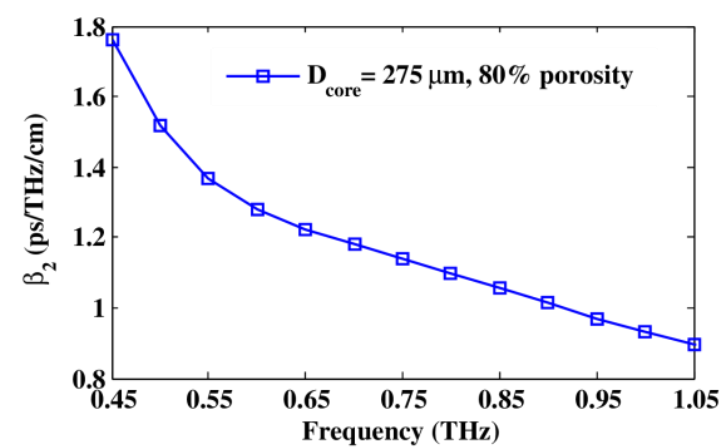

Fig. 6. Dispersion as a function of frequency at $D_{\text {core }}=275 \mu \mathrm{m}$ and $80 \%$ porosity.

The dispersion of the proposed fiber with varying operating frequency is shown in Fig. 6. As illustrated from the figure, dispersion decreases with increased frequency. The proposed fiber shows low dispersion variation in the entire band of interest. In the frequency band from 0.65-1.05 THz, we have found dispersion variation about
$1.05 \pm 0.22 \mathrm{ps} / \mathrm{THz} / \mathrm{cm}$. This flat and low dispersion is particularly suitable for broadband signal transmission over a long distance.

It should be noted that the mode field distributions shown in Fig. 2 consist of a series of dots inside the airholes, which may create difficulties in generating a beam with a high overlap. This could increase the coupling loss by leaking the mode power towards the cladding region. Quantum-cascade lasers (QCLs) have been considered as a convenient and potential source, which can radiate below $1 \mathrm{THz}$. A QCL based on metal-metal waveguides (MM) operates far from the optimum coupling condition. Besides, such QCLs suffer from a beam divergence issue that increases the coupling loss [14]. On the other hand, a QCL based on semi-insulating surface-plasmon (SI-SP) waveguides provides high output power, high efficiency and less divergent beam [15]. Therefore, in terms of coupling efficiency, an SI-SP based QCL can be used as a $\mathrm{THz}$ source for the proposed fiber.

In summary, we propose a new type of PC-PCF for efficient transmission of broadband $\mathrm{THz}$ waves. A thorough numerical analysis shows that it is possible to obtain an extremely low EML of $0.169 \mathrm{~dB} / \mathrm{cm}$ at 0.75 THz. Moreover, single mode propagation, power fraction and dispersion properties are also discussed rigorously. Considering the excellent guiding characteristics, the proposed fiber can be regarded as a good waveguide for $\mathrm{THz}$ guidance.

\section{References}

[1] J. J. Bai, J. N. Li, H. Zhang, H. Fang, S. J. Chang, Appl. Phys. B 103, 2 (2011).

[2] S. Atakaramians, S. Afshar, B. M. Fischer, D. Abbott, T. M. Monro, Opt. Express 16, 12 (2008).

[3] K. Wang, D. M. Mittleman, Nature 432, 7015 (2004).

[4] R. Islam, G. K. M. Hasanuzzaman, M. S. Habib, S. Rana, M. A. G. Khan, Opt. Fiber Technl. 24, (2015).

[5] M. I. Hasan, S. M. A. Razzak, G. K. M. Hasanuzzaman, M. S.Habib, IEEE Photon. Technol. Lett. 26, 23 (2014).

[6] S. F. Kaijage, Z. Ouyang, X. Jin, IEEE Photon. Technol. Lett. 25, 15 (2013).

[7] M. R. Hasan, M. A. Islam, A. A. Rifat, J. Eur. Opt. Soc. Rapid Publ. 12, 1 (2016).

[8] M. R. Hasan, M. A. Islam, M. S. Anower, S. M. A. Razzak, Appl. Opt. 55, 30 (2016).

[9] S. Ali et al. in $9^{\text {th }}$ Int. Conf. on Elect. and Comp. Eng., IEEE, (2016). doi: 10.1109/ICECE.2016.7853869

[10] K. Nielsen, H. K. Rasmussen, A. J. Adam, P. C. Planken, O. Bang, P. U. Jepsen, Opt. Express 17, 10 (2009).

[11] A. W. Snyder, J. D. Love, Optical waveguide theory (London, Chapman \& Hall 1983).

[12] L. Vincetti, A. Polemi, in Antennas and Propagation Society International Symposium, IEEE (2009)

[13] G. P. Agrawal, Nonlinear fiber optics (Boston, Academic Press 1989).

[14] B. S. Williams, Nat. Photon. 1, 9 (2007).

[15] H. W. Hubers et al., Opt. Express 13, 15 (2005). 